\title{
High prevalence of exposure to the child welfare system among street-involved youth in a Canadian setting: implications for policy and practice
}

Brittany Barker ${ }^{1,2+}$, Thomas Kerr ${ }^{1,3+}$, Gerald Taiaiake Alfred ${ }^{4}$, Michelle Fortin ${ }^{5}$, Paul Nguyen ${ }^{1}$, Evan Wood ${ }^{1,3}$ and Kora DeBeck ${ }^{1,2^{*}+}$

\begin{abstract}
Background: Street-involved youth are more likely to experience trauma and adverse events in childhood; however, little is known about exposure to the child welfare system among this vulnerable population. This study sought to examine the prevalence and correlates of being in government care among street-involved youth in Vancouver, Canada.

Methods: From September 2005 to November 2012, data were collected from the At-Risk Youth Study, a prospective cohort of street-involved youth aged 14-26 who use illicit drugs. Logistic regression analysis was employed to identify factors associated with a history of being in government care.

Results: Among our sample of 937 street-involved youth, 455 (49\%) reported being in government care at some point in their childhood. In a multivariate analysis, Aboriginal ancestry (adjusted odds ratio [AOR] $=2.07 ; 95 \%$ confidence interval [CI]: $1.50-2.85)$, younger age at first "hard" substance use (AOR $=1.10 ; 95 \% \mathrm{Cl}: 1.05-1.16)$, high school incompletion (AOR $=1.40 ; 95 \% \mathrm{Cl}: 1.00-1.95)$, having a parent that drank heavily or used illicit drugs (AOR $=1.48 ; 95 \%$ $\mathrm{Cl}: 1.09-2.01)$, and experiencing physical abuse (AOR $=1.90 ; 95 \% \mathrm{Cl}: 1.22-2.96)$ were independently associated with exposure to the child welfare system.
\end{abstract}

Conclusions: Youth with a history of being in government care appear to be at high-risk of adverse illicit substance-related behaviours. Evidence-based interventions are required to better support vulnerable children and youth with histories of being in the child welfare system, and prevent problematic substance use and street-involvement among this population.

Keywords: Child welfare system, Foster care, Government care, Street-involved youth, Youth substance use

\section{Background}

Children who are taken into government care, such as orphanages, foster homes, group homes, or otherwise become a ward of the state, constitute a highly vulnerable population. Although the transition into government care is intended to provide a safer and more stable environment, data indicates that many youth continue to struggle emotionally, physically,

\footnotetext{
*Correspondence: uhri-kd@cfenet.ubc.ca

${ }^{\dagger}$ Equal contributors

'British Columbia Centre for Excellence in HIV/AIDS, St. Paul's Hospital, 608-1081 Burrard Street, Vancouver BC V6Z 1Y6, Canada

${ }^{2}$ School of Public Policy, Simon Fraser University, Vancouver, Canada

Full list of author information is available at the end of the article
}

academically, and behaviourally during and after care [1-7]. In the Canadian province of British Columbia, the ministry responsible for child welfare (Ministry of Children and Family Development) estimated that in 2010/2011, 49.9\% of all youth in continuing government care were in special education classes and $63.8 \%$ were of Aboriginal ancestry [8]. These youth were also more likely to have intensive behavioural problems, serious mental illness, physical disabilities, and chronic health impairments [8].

The longer-term trajectories for children and youth who are taken into government care further underscores the vulnerability of this population. The struggle for 
youth exposed to the child welfare system continues well into early adulthood with elevated rates of poverty, underemployment, housing instability, incarceration, unplanned pregnancies and subsequent government involvement with parenting, mental health, physical health and substance use issues [5-7,9-13]. Given this profile, youth with a history of being in government care may be at greater risk for substance misuse and street-involvement. As such, we sought to examine the prevalence and correlates of youth with a history of being in government care among a cohort of street-involved youth who use illicit drugs in Vancouver, Canada.

\section{Methods}

Data for this cross-sectional study was collected at baseline study visits between September 2005 and November 2012, from the At-Risk Youth Study (ARYS), a prospective cohort of street-involved youth in Vancouver, Canada. Youth were eligible if they were between the ages of 14-26 at time of enrolment, had used illicit "hard" drugs in the past 30 days (e.g. crack, cocaine, heroin, or crystal methamphetamine) and provided written informed consent. The study has been described in detail elsewhere [14]; however, in brief, recruitment was undertaken using a snowball sampling approach with extensive outreach efforts in order to maximise the representativeness of this sample. In short, at baseline and subsequent semi-annual follow-up interviews, participants complete an interviewer-administered questionnaire and provide blood samples for $\mathrm{HIV}$ and $\mathrm{HCV}$ serology. The survey includes items on sociodemographic information, substance use patterns, sexual and drug-related risk behaviours, interactions with the criminal justice system, and engagement with health and social services. At each study visit, participants receive a $\$ 20$ monetary compensation (Canadian dollars) and the research ethics board of the University of British Columbia has approved the study.

The main outcome examined in the current analysis was a history of being in government care. This was defined as responding "yes" to the question: "As a child, did you ever live in an orphanage, a foster home, a group home, as a ward of the state, or away from your parents for a month or more (not including vacations)?" The comparison group was youth who reported not having been exposed to the child welfare system.

Explanatory variables thought to be potentially associated with our outcome of interest based on prior studies examining transitions in and out of government care $[12,13,15-17]$, including the following sociodemographic data: gender (female vs. male); Aboriginal ancestry (selfidentified as First Nations, Inuit, Métis vs. other); high school incompletion (yes vs. no); having a parent that drank heavily or used illicit drugs during their childhood (yes. vs. no); homelessness, defined as having no fixed address, sleeping on the street, couch surfing, or staying in a shelter or hostel in the last six months (yes vs. no); and living in the Downtown Eastside (DTES) in the last six months, defined as living in Vancouver's substance use epicenter and poorest neighborhood (yes vs. no). Behavioural and substance use variables, based on activities in the last six months, included: injection substance use (yes vs. no); drug overdose (yes vs. no) defined as having an adverse reaction as a result of consuming too much of a drug; daily injection or non-injection heroin use (yes vs. no), defined as any daily heroin use, regardless of the mode of administration (e.g., smoked, snorted, injected, swallowed, etc.); daily injection or non-injection cocaine use (yes vs. no); daily crack cocaine smoking (yes vs. no); daily injection or non-injection crystal methamphetamine use (yes vs. no); syringe sharing, defined as borrowing or lending used syringes (yes vs. no); engaging in sex work defined as exchanging sex for money, shelter, drugs or other commodities (yes vs. no); and participation in drug dealing (yes vs. no). Other factors included: age at first hard substance use, defined as the age participants first used non-injection crack, cocaine, heroin, or crystal methamphetamine (per year younger); testing positive for Hepatitis $C$ virus (yes vs. no); incarceration, defined as being in detention, prison, or jail overnight or longer in the previous six months (yes vs. no); having ever been the victim of sexual abuse (yes vs. no); having ever been the victim of physical abuse (yes vs. no); and recently experiencing an act of violence, defined as being attacked, assaulted, or suffering violence in the previous six months (yes vs. no).

In the bivariate analysis, dichotomous variables were analysed using Pearson's chi-square test and continuous variables were analysed using the Mann-Whitney test. To evaluate factors independently associated with our outcome of interest, all variables with $\mathrm{p}$-values that were $p<0.1$ in bivariate analyses were considered in a multivariate logistic regression. A backward model selection procedure was used to identify the multivariate model with the best overall fit, as indicated by the lowest Akaike Information Criterion [AIC] value [18]. All statistical analyses were performed using SAS software version 9.3 [SAS, Cary, NC]. All p-values are two sided.

\section{Results}

Over our study period, 937 youths enrolled in the ARYS cohort who provided complete responses to the questionnaire items were included in this analysis. In total, $91(8.6 \%)$ participants were excluded from the original sample of 1028 for not providing complete answers to the questions included in the analysis. Among this sample, 
292 (31\%) were female, 224 (24\%) were of Aboriginal ancestry, and the median age was 21.0 (interquartile range [IQR]: 20.0 - 23.0). In total, 455 (49\%) participants reported being in government care at some point in their childhood.

The characteristics of the study sample stratified by having a history of being in government care in childhood, along with the bivariate and multivariate analyses of factors associated with childhood exposure to government care are presented in Table 1 . In multivariate logistic regression factors that remained independently associated with our outcome of interest included: Aboriginal ancestry (adjusted odds ratio [AOR]: 2.07, 95\% confidence interval $[\mathrm{CI}]: 1.50-2.85)$, younger age at first hard substance use (AOR: 1.10, 95\% CI: 1.05 1.16), high school incompletion (AOR: 1.40, 95\% CI: $1.00-1.95)$, having a parent that drank heavily or used illicit drugs (AOR: 1.48, 95\% CI: 1.09 - 2.01), and being a victim of physical abuse (AOR: 1.90, 95\% CI: $1.22-2.96$ ).

\section{Discussion}

In the present study of street-involved youth in Vancouver, Canada, we found that experiences of being in government care in childhood were common, especially for youth who were of Aboriginal ancestry, victims of physical abuse, had not completed high school, had parents that drank heavily or used illicit substances, and had initiated first hard substance use at an earlier age. In Canada roughly $0.3 \%$ of youth have been exposed to the child welfare system [19], suggesting that street-involved youth who use substances are over 160 times more likely to have been in government care compared to the general population of youth.

Some of our findings are consistent with previous research. For example, youth exposed to the child welfare system have been found to be more likely to engage in risky behaviours and substance use later in life versus their peer group $[1,7,13]$. One US based study found higher intensity substance use patterns among homeless youth with a history of care compared to homeless youth without a history of care [9]. Similarly, our study found that youth exposed to child welfare were significantly more likely to initiate hard substance use at an earlier age. While we did not detect any other differences in substance use patterns among those with a history of being in government care, this may be attributed in part to the composition of our sample, which is restricted to high-risk youth that already engage in hard substance use. While it is hard to delineate whether the events in a youth's life preceding government intervention, or, the experiences during their tenure in care have the greatest influence and impact on risk of illicit substance use and becoming street-involved later in life, it is evident that more interventions are necessary to support these children and youth in avoiding substance use and street-involvement.

Youth in our sample who were formerly in government care were twice as likely to be of Aboriginal ancestry, which is consistent with government data $[8,19]$. The Aboriginal youth population represents approximately five percent of the total youth population in Canada; however, they account for approximately $50 \%$ of the children and youth in government care [19]. Previous research has identified the overrepresentation of ethnic minorities in child welfare services across high income nations [20-24]. Community organisations and researchers estimate there are three times as many Aboriginal children in the custody of the Canadian government today than there were during the time of residential schools - the government-funded, Church administered network of culturally destructive and harmful boarding schools that illegally removed thousands of Aboriginal children from their homes predominantly from the late $1800 \mathrm{~s}$ to the 1930s $[21,23,25]$. The majority of interventions in Aboriginal child welfare are due to charges of neglect, often in the context of perpetual poverty, inadequate housing, food insecurity, parental substance use, and other remnants of colonisation which continue to exacerbate the difficulties many Aboriginal parents face [25]. These and the intergenerational trauma experienced by families and communities as a result of losing more than a generation's example of parental-modeling has been associated with the high prevalence of neglect charges [21,25-27].

Interventions in Aboriginal child welfare should be driven by the respective community they affect, with First Nations and Aboriginal communities directing solutions that build-on their unique strengths and cultures. The proliferation of on-and-off reserve Aboriginal child welfare organisations in recent years has demonstrated some success in keeping children and youth tied to their communities and culture [28]. Early research indicates that Aboriginal children experience fewer emotional problems when care arrangements keep them tied to their culture and community [29,30]. Indigenous scholars and activists have called for the relationship between community development and child wellbeing to be examined, while continuing to increase culturally-based programs focused on prevention rather than intervention $[21,25,26,30,31]$. In addition, strengthening kinship care by providing appropriate and equitable support to Aboriginal relatives who assume custody of youth in care would likely help these youth remain tied to their communities and culture. Being cared for by relatives has been shown to provide an element of stability and improved outcomes for youth, although typically little support and funding from governments is provided to kinship caregivers, making it difficult for relatives to take on the financial burden of raising a 
Table 1 Baseline, bivariate and multivariable analyses of factors associated with a history of being in government care among street-involved youth in Vancouver, Canada $(\mathbf{n}=937)$

\begin{tabular}{|c|c|c|c|c|c|c|}
\hline \multirow{3}{*}{ Characteristic } & \multicolumn{3}{|c|}{ Government care exposure } & \multirow{3}{*}{$p$-value } & \multirow{3}{*}{$\begin{array}{l}\text { Adjusted odds } \\
\text { ratio, }\left(95 \% \mathrm{Cl}^{\mathrm{a}}\right)\end{array}$} & \multirow{3}{*}{$p$-value } \\
\hline & Yes & No & Odds ratio, & & & \\
\hline & $\overline{n=455, n(\%)}$ & $\overline{n=482 n(\%)}$ & & & & \\
\hline Age at first substance use & $15^{\mathbf{b}}$ & $16^{\mathbf{b}}$ & $1.12(1.06-1.18)$ & $<0.001$ & $1.10(1.05-1.16)$ & $<0.001$ \\
\hline Per year younger & $(13-16)^{c}$ & $(14-17)^{c}$ & & & & \\
\hline \multicolumn{7}{|l|}{ Gender } \\
\hline Female vs. Male & $157(35)$ & $135(28)$ & $1.35(1.03-1.79)$ & 0.032 & & \\
\hline \multicolumn{7}{|l|}{ Aboriginal ancestry } \\
\hline Yes vs. No & $139(31)$ & $85(18)$ & $2.05(1.51-2.80)$ & $<0.001$ & $2.07(1.50-2.85)$ & $<0.001$ \\
\hline \multicolumn{7}{|l|}{ High school incompletion } \\
\hline Yes vs. No & $375(82)$ & $346(72)$ & $1.84(1.35-2.52)$ & $<0.001$ & $1.40(1.00-1.95)$ & 0.049 \\
\hline \multicolumn{7}{|c|}{ Parental alcohol/ Substance use } \\
\hline Yes vs. No & $354(78)$ & $320(66)$ & $1.77(1.33-2.37)$ & $<0.001$ & $1.48(1.09-2.01)$ & 0.012 \\
\hline \multicolumn{7}{|l|}{ Lives in the DTES ${ }^{d}$} \\
\hline Yes vs. No & $124(27)$ & $139(29)$ & $0.92(0.70-1.23)$ & 0.590 & & \\
\hline \multicolumn{7}{|l|}{ Homeless $^{d}$} \\
\hline Yes vs. No & $349(77)$ & $352(73)$ & $1.22(0.90-1.64)$ & 0.200 & & \\
\hline \multicolumn{7}{|l|}{ Injection substance use ${ }^{d}$} \\
\hline Yes vs. No & $132(29)$ & $150(31)$ & $0.91(0.68-1.20)$ & 0.482 & & \\
\hline \multicolumn{7}{|l|}{ Daily heroin use $e^{d, e}$} \\
\hline Yes vs. No & $49(11)$ & $68(14)$ & $0.74(0.50-1.09)$ & 0.123 & & \\
\hline \multicolumn{7}{|l|}{ Daily cocaine use $e^{e}$} \\
\hline Yes vs. No & $19(4)$ & $21(4)$ & $0.96(0.51-1.80)$ & 0.891 & & \\
\hline \multicolumn{7}{|l|}{ Daily crack use ${ }^{d}$} \\
\hline Yes vs. No & $85(19)$ & $87(18)$ & $1.04(0.75-1.45)$ & 0.803 & & \\
\hline \multicolumn{7}{|l|}{ Daily crystal meth use ${ }^{e}$} \\
\hline Yes vs. No & $72(16)$ & $54(11)$ & $1.49(1.02-2.18)$ & 0.039 & & \\
\hline \multicolumn{7}{|l|}{ Drug overdose ${ }^{d}$} \\
\hline Yes vs. No & $58(13)$ & $46(10)$ & $1.39(0.92-2.09)$ & 0.120 & & \\
\hline \multicolumn{7}{|l|}{ Hepatitis C positive } \\
\hline Yes vs. No & $90(20)$ & $64(13)$ & $1.61(1.14-2.29)$ & 0.008 & $1.36(0.94-1.97)$ & 0.099 \\
\hline \multicolumn{7}{|l|}{ Syringe sharing ${ }^{d}$} \\
\hline Yes vs. No & $38(8)$ & $37(8)$ & $1.10(0.68-1.76)$ & 0.703 & & \\
\hline \multicolumn{7}{|l|}{ Victim of violence $^{d}$} \\
\hline Yes vs. No & $214(47)$ & $210(44)$ & $1.15(0.89-1.49)$ & 0.287 & & \\
\hline \multicolumn{7}{|l|}{ Incarcerated $^{d}$} \\
\hline Yes vs. No & $96(21)$ & $86(18)$ & $1.23(0.89-1.70)$ & 0.208 & & \\
\hline \multicolumn{7}{|l|}{ Physical abuse } \\
\hline Yes vs. No & $413(91)$ & $397(82)$ & $2.11(1.42-3.12)$ & $<0.001$ & $1.90(1.22-2.96)$ & 0.005 \\
\hline \multicolumn{7}{|l|}{ Sexual abuse } \\
\hline Yes vs. No & $345(76)$ & $317(66)$ & $1.63(1.23-2.17)$ & $<0.001$ & $1.29(0.93-1.78)$ & 0.124 \\
\hline Sex work ${ }^{d}$ & & & & & & \\
\hline Yes vs. No & $54(12)$ & $43(9)$ & $1.38(0.90-2.10)$ & 0.140 & & \\
\hline Drug dealing $^{d}$ & & & & & & \\
\hline Yes vs. No & $254(56)$ & $255(53)$ & $1.13(0.87-1.46)$ & 0.370 & & \\
\hline
\end{tabular}


youth [32-35]. Our study findings indicate that continued attention and efforts to improve outcomes for vulnerable Aboriginal youth and to address their overrepresentation in the child welfare system are required.

Our study found that having a parent that drank heavily or used illicit substances was associated with a history of being in government care. Previous research has demonstrated a relationship between parental substance use and subsequent child welfare involvement, predominantly in child neglect cases $[21,36,37]$. One study that reviewed 639 child welfare cases in the US found that $79 \%$ of caregivers involved were misusing at least one substance [16]. Similarly, the 'Canadian Incidence Study of Reported Child Abuse and Neglect' found that substance use was the most frequent root problem in caregiver-related cases [38]. These studies, along with our research, suggest a potential pathway between parental substance use, elevated risk for child maltreatment and subsequent out-of-home placements. This indicates that efforts to address parental substance use are needed. This may involve increasing access to evidence-based addiction treatments, parenting supports and other relevant social services.

Exposure to the child welfare system was also associated with physical abuse among our sample. This is supported by prior studies which indicate that youth in care are more likely than the general population to have experienced parental neglect, domestic violence, physical, and sexual abuse $[1,3,7]$. Given these findings, targeted supports to aid these youth cope with their traumatic experiences appears to be particularly warranted.

High school incompletion was another factor found to be associated with a history of being in government care. Low educational attainment among youth in government care is consistent with a wealth of research that indicates this population is much more likely to fail a grade, not graduate, have lower standardised test scores and lower post secondary education achievement levels [8,17,39-41]. When youth who have not completed high school are emancipated from government care, and do not have familial or financial support, the transition into financial independence can be unsuccessful and lead to instability. Research has found that low or a lack of educational attainment for youth in government care results in elevated rates of reliance on social assistance and many experiencing acute poverty $[6,7,10]$. Early interventions for youth currently in government care are needed to ensure a trajectory that follows and supports students through to high school completion in order to mitigate some of the poor outcomes identified.

Together these study findings highlight that youth with a history of being in government care are significantly more susceptible to substance use and streetinvolvement later in life, suggesting that action is needed on multiple fronts. For families on the cusp of having their children taken into government custody, parental support and training for parents has been associated with creating healthy families [15,42-44]. Given the association between parental substance use and youth exposure to the child welfare system, a focus on addiction treatment within parental support programs appears to be particularly relevant. For children and youth who are in government care and do not have a safe family environment to return to, evidence suggests that actions to identify permanent care options quickly and efficiently are advantageous [5,45-49]. Also needed are efforts to ensure that appropriate supports and services are in place after youth are emancipated from care, or 'age-out' of the system. Independent living programs are initiatives that focus on life skills training for at-risk youth and involve assistance with setting up bank accounts, finding housing and employment, goal setting, developing healthy relationships, and conflict resolution. A small number of studies have found these programs to be associated with improved outcomes for youth in government care [5,6]; however, other studies have reported mixed results [50,51], indicating that more research is needed to ensure that newly emancipated youth are effectively supported by services and are able to better transition to independence.

This study has several limitations. First, as with all community-recruited research cohorts, the ARYS cohort is not a random sample and therefore may not generalise to other populations of street youth. Second, data collected was based on self report and thus could be subject to response biases, including socially desirable responding, which may have resulted in under reporting of illicit substance use and other stigmatised behaviours. As a result, the prevalence of some risk behaviours may have been underestimated in the present study. However, self reported risk behaviour has been shown to be largely accurate among adult substance-using populations [52] and also among various youth populations [53]. Furthermore, we acknowledge that there may be some unmeasured risk (e.g., assaults or traumatic events that occurred outside of time spent in child protection) or other confounding factors (e.g., length of time in care, multiple placements) that were not considered in our analyses. With respect to how we currently measure exposure to the child welfare system, the binary nature of participant response (yes vs. no) limits the interpretation of the findings. Specifically, important information concerning exposure to the child welfare system (e.g., number of placements, length of time in care) was not captured by our study instrument, including the various factors that have been shown to have a negative impact on longterm outcomes. For example, multiple care placements have been associated with negative impacts upon 
emotional and cognitive development, behavioural issues, and life-skills development $[5,46,48,49,54,55]$. Given that the intensity and frequency of exposure were not measured in this analysis, we recognise future analyses should seek to measure these important aspects of child welfare system exposure.

\section{Conclusions}

Based on our study sample, street-involved youth in Vancouver are over 160 times more likely to have a history of being in government care compared to the general population of youth. Our study found that those with a history of being in government care were more likely to be of Aboriginal ancestry, have started using hard substances at an earlier age, have a history of physical abuse, have a parent that drank heavily or used illicit substances, and did not complete high school. Outcomes associated with the child welfare system have become a public health concern, and one that governments have failed to adequately address. However, these findings give policymakers potential areas for redress and demonstrate the need for interventions to support families and youth along the continuum of risk. This includes interventions to support at-risk families before government involvement is necessary, policies for children and youth currently in care, and services to help youth successfully transition out of care and into early adulthood.

\section{Competing interests}

The authors declare that they have no competing interests.

\section{Authors' contributions \\ The specific contributions of each author are as follows: BB, KD, TK and EW designed the study and wrote the protocol, BB managed the literature searches and $\mathrm{BB}$ and $\mathrm{KD}$ prepared the first draft of the analysis; PN conducted the statistical analyses with input from BB, KD; all authors contributed to the main content and provided critical comments on the final draft. All authors approved the final manuscript.}

\section{Acknowledgements \\ The authors thank the ARYS study participants for their contribution to the research, as well as current and past researchers and staff. We would specifically like to thank Cody Callon, Jennifer Matthews, Deborah Graham, Peter Vann, Steve Kain, Tricia Collingham, and Carmen Rock for their research and administrative assistance. The study was supported by the US National Institutes of Health [R01-DA028532] and the Canadian Institutes of Health Research [MOP-102742]. This research was undertaken, in part, thanks to funding from the Canada Research Chairs program through a Tier 1 Canada Research Chair in Inner City Medicine, which supports Dr. Evan Wood. Dr. Kora DeBeck is supported by a MSFHR/St. Paul's Hospital Foundation-Providence Health Care Career Scholar Award. Funding sources had no further role in study design; in the collection, analysis and interpretation of data; in the writing of the report; or in the decision to submit the paper for publication.}

\section{Author details}

${ }^{1}$ British Columbia Centre for Excellence in HIV/AIDS, St. Paul's Hospital, 608-1081 Burrard Street, Vancouver BC V6Z 1Y6, Canada. ²School of Public Policy, Simon Fraser University, Vancouver, Canada. ${ }^{3}$ Department of Medicine, University of British Columbia, Vancouver, Canada. ${ }^{4}$ Indigenous Governance, University of Victoria, Victoria, Canada. ${ }^{5}$ Watari Counselling \& Support Services Society, Vancouver, BC, Canada.
Received: 1 October 2013 Accepted: 18 February 2014

Published: 24 February 2014

\section{References}

1. Rutter M: Children in substitute care: some conceptual considerations and research implications. Child Youth Serv Rev 2000, 22:685-703.

2. Jonson-Reid M: Foster care and future risk of maltreatment. Child Youth Serv Rev 2003, 25:271-294.

3. Drapeau S, Saint-Jacques M-C, Lépine R, Bégin G, Bernard M: Processes that contribute to resilience among youth in foster care. J Adolesc 2007, 30:977-999.

4. Marquis R, Leshied D, O'Neill A: The relationship of child neglect and physical maltreatment to placement outcomes and behavioural adjustment in children in foster care: a Canadian perspective. Child Welfare 2008, 87:5-25.

5. Reilly T: Transition from care: status and outcomes of youth who age out of foster care. Child Welfare 2003, 82:727-746.

6. Collins ME: Transition to adulthood for vulnerable youths: a review of research and implications for policy. Soc Serv Rev 2001, 75:271-291.

7. Mendes P: Graduating from the child welfare system: a case study of the leaving care debate in Victoria, Australia. J Soc Work 2005, 5:155-171.

8. Ministry of Children and Family Development [MCFD]: Educational Experiences of Children Under a Continuing Custody Order. Victoria: Ministry of Children and Family Development; 2012.

9. Hudson A, Nandy K. Comparisons of substance abuse, high-risk sexual behaviour and depressive symptoms among homeless youth with and without a history of foster care placement. Contemp Nurse 2012, 42:178-186.

10. Mendes P, Moslehuddin B: Graduating from the child welfare system: a comparison of the UK and Australian leaving care debates. Int I Soc Welf 2004, 25:332-339.

11. Kushel M, Yen I, Gee L, Courtney M: Homelessness and health care access after emancipation. Arch Pediatr Adolesc Med 2007, 161:986-993.

12. Fowler PJ, Toro PA, Miles BW: Pathways to and from homelessness and associated psychosocial outcomes among adolescents leaving the foster care system. Am J Public Health 2009, 99:1453-1458.

13. Courtney M, Piliavin I, Grogan-Kaylor A, Nesmith A: Foster youth transitions to adulthood: a longitudinal view of youth leaving care. Child Welfare 2001, 80:685-717.

14. Wood E, Stoltz J-A, Montaner JS, Kerr T: Evaluating methamphetamine use and risks of injection initiation among street youth: the ARYS study. Harm Reduct J 2006, 3:18-24.

15. Barth R: On their own: the experiences of youth after foster care. Child Adolesc Soc Work 1990, 7:419-440.

16. Besinger B, Garland A, Litrownik A, Landsverk J: Caregiver substance abuse among maltreated children placed in out-of-home care. Child Welfare 1999, 78:221-239.

17. Cox T: Improving educational outcomes for children and youths in foster care. Child Sch 2013, 35:59-62.

18. Akaike $\mathrm{H}$ : A new look at the statistical model identification. IEEE Transactions on Automatic Control 1974, 19:716-723.

19. Statistics Canada: 2011 National Household Survey: Aboriginal Peoples in Canada: First Nations People, Métis and Inuit. [http://www.statcan.gc.ca/dailyquotidien/130508/dq130508a-eng.pdf]

20. Fluke JD, Yuan Y-YT, Hedderson J, Curtis PA: Disproportionate representation of race and ethnicity in child maltreatment: investigation and victimization. Child Youth Serv Rev 2003, 25:359-373.

21. Trocmé N, Knoke D, Blackstock C: Pathways to the overrepresentation of aboriginal children in Canada's child welfare system. Soc Serv Rev 2004, 78:577-600.

22. Tilbury C: The over-representation of indigenous children in the Australian child welfare system. Int J Soc Welf 2008, 18:57-64.

23. Blackstock C: The Canadian human rights tribunal on first nations child welfare: why if Canada wins, equality and justice lose. Child Youth Serv Rev 2011, 33(1):187-194.

24. Villegas S, Rosenthal JA, O'Brien K, Pecora P: Health outcomes for adults in family foster care as children: an analysis by ethnicity. Child Youth Serv Rev 2011, 33:110-117.

25. Blackstock C, Trocmé N: Community-based child welfare for Aboriginal children: supporting resilience through structural change. Soc Policy J N Z 2005, 24:12-33. 
26. Kirmayer L, Simpson C, Cargo M: Healing traditions: culture, community and mental health promotion with Canadian Aboriginal peoples. Australas Psychiatr 2003, 11:15-23.

27. Kirmayer L, Valaskakis GG: Healing Traditions: The Mental Health of Aboriginal Peoples in Canada. British Columbia: University of British Columbia Press; 2009

28. Blackstock C, Trocmé N, Bennett M: Child maltreatment investigations among Aboriginal and non-aboriginal families in Canada. Violence Against Wom 2004, 10:901-916.

29. Carriere J: Connectedness and health for First Nation adoptees. Paediatr Child Health 2005, 10:545.

30. Carriere J: Promising practice for maintaining identities in First Nation adoption. First Peoples Child and Family Review 2007, 3:46-64.

31. Carter VB: Factors predicting placement of urban American Indian/Alaskan Natives into out-of-home care. Child Youth Serv Rev 2010, 32:657-663.

32. Berrick JD, Barth RP, Needell B: A comparison of kinship foster homes and foster family homes: implications for kinship foster care as family preservation. Child Youth Serv Rev 1994, 16:33-63.

33. Courtney ME, Barth RP: Pathways of older adolescents out of foster care: implications for independent living services. Soc Work 1996, 41:75-83.

34. Rubin DM, Downes KJ, O'Reilly ALR, Mekonnen R, Luan X, Localio R: Impact of kinship care on behavioural well-being for children in out-of-home care. Arch Pediatr Adolesc Med 2008, 162:550-556.

35. Sakai C, Lin H, Flores $\mathrm{G}$ : Health outcomes and family services in kinship care analysis of a national sample of children in the child welfare system. Arch Pediatr Adolesc Med 2011, 165:159-165.

36. Barth R: Preventing child abuse and neglect with parent training: evidence and opportunities. Futur Child 2009, 19:95-118.

37. Semidei J, Radel L, Nolan C: Substance abuse and child welfare: clear linkages and promising responses. Child Welfare 2001, 80:109-128.

38. Trocmé N, Maclaurin B, Fallon B, Daciuk J, Billingsley D, Tourigny M, Mayer M, Wright J, Barter K, Burford G, Hornick J, Sullivan R, McKenzie B: Canadian incidence study of reported child abuse and neglect. Ottawa: Health Canada; 2012.

39. Fowler PJ, Toro PA, Miles BW: Emerging adulthood and leaving foster care: settings associated with mental health. Am J Community Psychol 2011, 47:335-348

40. Conger D, Finkelstein M: Foster care and school mobility. J Negro Educ 2003, 72:91-103.

41. Zetlin A, Weinberg L, Kimm C: Improving education outcomes for children in foster care: intervention by an education liaison. J Educ Stud Placed Risk 2009, 9:421-429.

42. Clulow C, Donaghy M: Developing the couple perspective in parenting support: evaluation of a service initiative for vulnerable families. $J$ Fam Ther 2010, 32:142-168.

43. Halpenny AM: Parenting and family support for families "at risk" - implications from child abuse reports. Ir J App/ Soc Stud 2011, 12:89-98.

44. Thomlison B: Characteristics of evidence-based child maltreatment interventions. Child Welfare 2003, 82:541-569.

45. Kufeldt K, Armstrong J, Dorosh M: How children in care view their own and their foster families: a research study. Child Welfare 1995, 74:695-715.

46. Harden B: Safety and stability for foster children: a developmental perspective. Futur Child 2004, 14:30-47.

47. Fisher $P$, Burraston $B$, Pears K: The early intervention foster care program: permanent placement outcomes from a randomized trial. Child Maltreat 2005, 10:61-71.

48. Unrau YA, Seita JR, Putney KS: Former foster youth remember multiple placement moves: a journey of loss and hope. Child Youth Serv Rev 2000 30:1256-1266

49. Dregan A, Gulliford MC: Foster care, residential care and public care placement patterns are associated with adult life trajectories: population-based cohort study. Soc Psychiatry Psychiatr Epidemiol 2012, 47:1517-1526.

50. Lemon $\mathrm{K}$, Hines AM, Merdinger J: From foster care to young adulthood: the role of independent living programs in supporting successful transitions. Child Youth Serv Rev 2005, 27:251-270.

51. Montgomery P, Donkoh C, Underhill K: Independent living programs for young people leaving the care system: the state of the evidence. Child Youth Serv Rev 2006, 28:1435-1448.

52. Darke S: Self-report among injecting drug users: a review. Drug Alcohol Depend 1998, 51:253-263.
53. Brener ND, Billy JOG, Grady WR: Assessment of factors affecting the validity of self-reported health-risk behaviour among adolescents: evidence from the scientific literature. J Adolesc Health 2003, 33:436-457.

54. Newton RR, Litrownik AJ, Landsverk JA: Children and youth in foster care: disentangling the relationship between problem behaviours and number of placements. Child Abuse Negl 2000, 24:1363-1374.

55. Price JM, Chamberlain P, Landsverk J, Reid JB, Leve LD, Laurent H: Effects of a foster parent training intervention on placement changes of children in foster care. Child Maltreat 2008, 13:64-75.

doi:10.1186/1471-2458-14-197

Cite this article as: Barker et al:: High prevalence of exposure to the child welfare system among street-involved youth in a Canadian setting: implications for policy and practice. BMC Public Health 2014 14:197

\section{Submit your next manuscript to BioMed Central and take full advantage of:}

- Convenient online submission

- Thorough peer review

- No space constraints or color figure charges

- Immediate publication on acceptance

- Inclusion in PubMed, CAS, Scopus and Google Scholar

- Research which is freely available for redistribution 